\title{
Ansiedade e depressão durante o estado de emergência nacional por COVID-19: realidade numa Unidade de Saúde Familiar
}

Marta Marques Santana, ${ }^{1}$ Ana Margarida Gonçalves, ${ }^{1}$ Daniela Henriques, ${ }^{1}$ Denise Velho, ${ }^{1}$ João Magalhães Cardoso, ${ }^{1}$ Joana Antunes, ${ }^{1}$ Mariana Coimbra ${ }^{1}$

\section{RESUMO}

Objetivos: Passaram alguns meses desde que foi confirmado o primeiro caso da doença COVID-19 em Portugal. A rápida progressão desta pandemia levou a diversas alterações no quotidiano dos utentes da Unidade de Saúde Familiar (USF) Santiago de Leiria, provavelmente com impacto na sua saúde mental. O objetivo deste trabalho é determinar os graus de ansiedade e depressão dos utentes da USF Santiago de Leiria durante o estado de emergência nacional.

Tipo de estudo: Estudo transversal descritivo com componente analítica.

Local: USF Santiago de Leiria.

População: Utentes inscritos na USF Santiago de Leiria.

Métodos: Estudo realizado através de um questionário online constituído por um inquérito sociodemográfico, a Escala Hospitalar de Ansiedade e Depressão e uma questão relativa ao apoio social, enviado a 1.000 utentes da USF em 9 de abril de 2020 e válido para submissão de respostas até 1 de maio de 2020.

Resultados: Dos 285 indivíduos que participaram, 47\% apresentavam sintomas de ansiedade e 32\% sintomas depressivos. Indivíduos do género feminino apresentaram níveis mais elevados quer de sintomatologia ansiosa quer de sintomatologia depressiva. Indivíduos com níveis mais baixos de escolaridade, desempregados e trabalhadores em lay-off apresentaram uma maior prevalência de ansiedade. Entre os inquiridos, 89,1\% referiu ter o apoio social necessário.

Conclusão: Este trabalho demonstra uma elevada prevalência dos sintomas de ansiedade e depressão, com maior expressão no sexo feminino. Desta premissa, novas estratégias de melhoria da saúde mental dos utentes poderão surgir e evitar ainda mais danos provocados pela COVID-19.

Palavras-chave: Ansiedade; COVID-19; Depressão; Pandemia; SARS-CoV-2.

\section{INTRODUÇÃO}

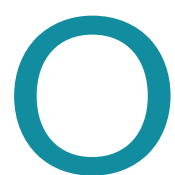

vírus SARS-CoV-2 foi identificado pela primeira vez em humanos em dezembro de 2019, na China. ${ }^{1}$ Desde então, o número de doentes infetados cresceu exponencialmente a nível mundial, sendo a Europa um dos conti-

1. Médico Interno de Medicina Geral e Familiar. USF Santiago de Leiria. Leiria, Portugal.

2. Médica Especialista de Medicina Geral e Familiar. USF Santiago de Leiria. Leiria, Portugal. nentes mais afetados por esta doença. ${ }^{2} \mathrm{~A} 11$ de março, a Organização Mundial da Saúde (OMS) declarou o surto de COVID-19 como pandemia. ${ }^{3}$

Em Portugal, o primeiro caso diagnosticado ocorreu a 2 de março de $2020 .{ }^{4}$ Com o objetivo de retardar a progressão do vírus foram encerradas escolas, recomendado o isolamento social e, mais tarde, a 18 de março, foi declarado o estado de emergência nacional. Este implicou uma adaptação da sociedade a novas regras e restrições, entre as quais o dever geral de recolhimento, o dever 
especial de proteção a pessoas com mais de 70 anos e a doentes crónicos, assim como o isolamento obrigatório de doentes com diagnóstico de COVID-19 ou em situação de vigilância ativa (monitorização diária pela autoridade de saúde durante o período máximo de incubação da doença aos contactos próximos de alto risco de um doente com COVID-19). ${ }^{5-6}$ Ao nível dos estabelecimentos públicos generalizou-se o teletrabalho, $o$ atendimento não presencial e o encerramento de empresas com atendimento público de serviços não essenciais. ${ }^{6}$

Esta decisão deveu-se ao facto de o vírus ter uma rápida transmissão através de gotículas respiratórias e do contacto direto com secreções infetadas. ${ }^{7}$ O SARS-CoV-2 está associado a um período de incubação médio de 5,1 dias (até um máximo de 14 dias), favorecendo o possível contágio por pessoas assintomáticas que não cumpram as recomendações. ${ }^{1}$ Os principais sintomas incluem febre, calafrios, tosse, coriza, odinofagia, dispneia, mialgias, náuseas, vómitos e diarreia. ${ }^{7}$ Os casos podem ser desde ligeiros a severos, com necessidade de internamento numa unidade de cuidados intensivos, com recurso a ventilação invasiva ou, até mesmo, levar à morte. ${ }^{8}$

A rápida progressão desta pandemia, o estado de isolamento social, a primeira morte, o sensacionalismo jornalístico, o desconhecido e a incerteza em relação ao futuro podem levar a sentimentos de angústia, medo de contrair a doença, de morrer e, até mesmo, estigma social. ${ }^{9-10}$ Por isso, os autores do presente estudo propuseram-se tentar compreender de que forma a saúde mental da comunidade da Unidade de Saúde Familiar (USF) Santiago de Leiria foi afetada durante o período inicial da pandemia, de forma a serem criados mecanismos de ajuda às pessoas mais vulneráveis, durante e após terminado o estado de emergência.

Neste contexto colocou-se a hipótese de poder ocorrer um aumento dos níveis de ansiedade e depressão na população estudada associada à pandemia. Desta forma, este estudo teve como objetivo principal determinar os graus de ansiedade e depressão dos utentes da USF Santiago de Leiria durante o estado de emergência nacional, assim como analisar a existência de associação entre os graus de ansiedade e depressão e as seguintes variáveis: sexo, idade, escolaridade, estado civil, situação laboral. Pretendeu-se ainda avaliar a existência de apoio, em caso de necessidade.

\section{MÉTODOS}

Foi realizado um estudo transversal descritivo com componente analítica de uma amostra aleatória dos 12.741 utentes da USF Santiago de Leiria em março de 2020. Com o intuito de se obter uma amostra representativa com intervalo de confiança de $95 \%$ foram enviados formulários de resposta anónima a 1.000 utentes da USF Santiago de Leiria, com idade igual ou superior a 18 anos e com endereço de correio eletrónico no Registo Nacional de Utentes. $\mathrm{O}$ acesso às listagens dos utentes foi efetuado através do Módulo de Informação e Monitorização das Unidades Funcionais (MIM@UF) e a aleatorização no Microsoft Excel ${ }^{\circledR}$. A aplicação do questionário decorreu entre 9 de abril e 1 de maio de 2020.

O questionário incluiu questões para caracterização sociodemográfica, a Escala Hospitalar de Ansiedade e Depressão (HADS) - versão portuguesa de McIntyre, Pereira, Soares, Gouveia e Silva (1999) e uma questão sobre a existência de apoio social.

No questionário sociodemográfico constavam o género, a idade, o estado civil, o nível de escolaridade e a situação profissional.

Quanto à escala HADS foi desenvolvida para avaliar, de forma muito breve, os níveis de ansiedade e depressão em doentes com patologia física em tratamento ambulatório. Apesar de este ter sido o seu objetivo inicial tem sido amplamente usada em investigação e na prática clínica. É uma escala de autoadministração e demora cerca de cinco minutos a ser preenchida. Inclui 14 itens (sete para avaliar a ansiedade e sete para avaliar a depressão). O modo de resposta varia de zero a três ( 0 = baixo e 3 = elevado $)$ numa escala de Likert. Para cada questão existem quatro possibilidades de resposta, sendo que a pessoa escolhe a que melhor se adapta ao que sentiu na última semana. Os resultados totais em cada subescala variam de 0 a 21 . A severidade da ansiedade e da depressão pode ser classificada como «normal» (de 0 a 7), «leve» (de 8 a 10), «moderada» (de 11 a 15) ou «severa» (de 16 a 21)..$^{11-12}$

A questão final pretendia aferir, de forma sucinta, a perceção do indivíduo da disponibilidade de apoio em caso de necessidade.

Foi criada uma base de dados em Microsoft Excel ${ }^{\circledR}$, com posterior exportação para o Software Statistical Package for Social Sciences (SPSS) ${ }^{\circledR}$ para a análise estatística. 


\begin{tabular}{|c|c|c|c|c|c|c|c|}
\hline & & Normal & Leve & Moderada & Severa & Total & \\
\hline \multirow{2}{*}{ Género } & Masculino & 61 & 18 & 17 & 1 & 97 & \multirow{2}{*}{$p=0,039^{*}$} \\
\hline & Feminino & 89 & 39 & 50 & 10 & 188 & \\
\hline \multicolumn{2}{|l|}{ Total } & 150 & 57 & 67 & 11 & 285 & \\
\hline
\end{tabular}

Legenda: ${ }^{*}=$ qui-quadrado.

\begin{tabular}{|c|c|c|c|c|c|c|c|}
\hline & & Normal & Leve & Moderada & Severa & Total & \\
\hline \multirow{2}{*}{ Género } & Masculino & 75 & 13 & 7 & 2 & 97 & \multirow{2}{*}{$p=0,09 *$} \\
\hline & Feminino & 120 & 35 & 33 & 0 & 188 & \\
\hline \multicolumn{2}{|l|}{ Total } & 195 & 48 & 40 & 2 & 285 & \\
\hline
\end{tabular}

Legenda: ${ }^{*}=$ qui-quadrado.

\begin{tabular}{|c|c|c|c|}
\hline Nível de ansiedade & Média & $n$ & \\
\hline Normal & 46,37 & 150 & \multirow{4}{*}{$p=0,918^{*}$} \\
\hline Leve & 47,07 & 57 & \\
\hline Moderada & 45,67 & 67 & \\
\hline Severa & 47,64 & 11 & \\
\hline Total & 46,39 & 285 & \\
\hline
\end{tabular}

Legenda: ${ }^{*}=$ ANOVA.

\begin{tabular}{|c|c|c|c|}
\hline Nível de depressão & Média & $n$ & \\
\hline Normal & 46,15 & 197 & \multirow{4}{*}{$p=0,742^{*}$} \\
\hline Leve & 46,15 & 48 & \\
\hline Moderada & 47,48 & 40 & \\
\hline Severa & 54,5 & 2 & \\
\hline Total & 46,39 & 285 & \\
\hline
\end{tabular}

Legenda: * = ANOVA.
A análise estatística consistiu na caracterização dos indivíduos respondentes, aplicando testes de inferência estatística, recorrendo a análise bivariada para testar a hipótese nula de não existir associação entre o grau de ansiedade e de depressão com as restantes variáveis, para um intervalo de confiança de $95 \%(\alpha=0,05)$. O procedimento estatístico foi realizado com recurso ao SPSS ${ }^{\oplus}$. Foram aplicados testes do qui-quadrado para as variáveis qualitativas e a ANOVA para a idade, única variável quantitativa em análise.

O consentimento informado foi garantido através da aposição de informações sobre o estudo no cabeçalho do questionário e da devolução voluntária dos questionários online. O protocolo do estudo foi aprovado pela comissão de ética da ARS Centro.

\section{RESULTADOS}

Dos 285 indivíduos que participaram no estudo, $66 \%$ era do género feminino. A média de idades foi de 46 anos (idade mínima de 19 e máxima de 82 anos). A maioria dos indivíduos da amostra era casada ou em união de facto $(71,9 \%)$, com licenciatura $(38,2 \%)$ e trabalhador por conta de outrem $(68,4 \%)$.

Aproximadamente $47 \%$ dos indivíduos apresentavam sintomas de ansiedade e $32 \%$ sintomas de depressão. Relativamente à ansiedade, $20 \%$ da amostra apresentava sintomas leves, $23,5 \%$ sintomas moderados e $3,9 \%$ sintomas severos de ansiedade. No que diz respeito à sintomatologia depressiva, $16,8 \%$ apresentava sintomatologia depressiva leve, $14 \%$ sintomatologia moderada e $0,7 \%$ sintomatologia severa.

Efetuando uma análise bivariada verifica-se que, em relação ao género, a ansiedade e a depressão foram mais prevalentes no género feminino, com significância estatística, como se constata nas Tabelas 1 e 2. No que concerne à ansiedade, cerca de $53 \%$ das mulheres e $37 \%$ dos homens apresentavam algum grau de ansiedade (leve a severa). Em relação à depressão, 36\% das 


\begin{tabular}{|c|c|c|c|c|c|c|c|}
\hline & & \multicolumn{5}{|c|}{ Nível de ansiedade } & \\
\hline & & Normal & Leve & Moderada & Severa & Total & \\
\hline \multirow{4}{*}{ Estado civil } & Solteiro & 24 & 6 & 9 & 1 & 40 & \multirow{4}{*}{$P=0,910^{*}$} \\
\hline & Casado/união de facto & 105 & 44 & 49 & 7 & 205 & \\
\hline & Divorciado & 20 & 7 & 9 & 3 & 39 & \\
\hline & Viúvo & 1 & 0 & 0 & 0 & 1 & \\
\hline Total & & 150 & 57 & 67 & 11 & 285 & \\
\hline
\end{tabular}

Legenda: ${ }^{*}=$ qui-quadrado.

\section{TABELA 6. Associação entre o estado civil e o nível de depressão.}

\begin{tabular}{|l|l|c|c|c|c|c|c|}
\multicolumn{2}{c|}{} & \multicolumn{6}{c|}{ Nível de depressão } \\
\cline { 3 - 7 } \multicolumn{2}{c|}{} & Normal & Leve & Moderada & Severa & Total & \\
\hline \multirow{3}{*}{ Estado civil } & Solteiro & 28 & 7 & 5 & 0 & 40 & \\
\cline { 2 - 8 } & Casado/união de facto & 140 & 37 & 28 & 0 & 205 & \multirow{3}{*}{$P=0,096^{*}$} \\
\cline { 2 - 8 } & Divorciado & 26 & 4 & 7 & 2 & 39 & \\
\cline { 2 - 8 } & Viúvo & 1 & 0 & 0 & 0 & 1 & \\
\hline Total & & 195 & 48 & 40 & 2 & 285 & \\
\hline
\end{tabular}

Legenda: * = qui-quadrado.

mulheres e $23 \%$ dos homens apresentavam algum grau de depressão (leve a severa).

Não se verificou associação entre a ansiedade ou a depressão e a idade (Tabelas 3 e 4).

Também não se verificou associação entre estas variáveis e o estado civil (Tabelas 5 e 6).

A Tabela 7 evidencia a associação estatisticamente significativa entre o nível de escolaridade e o nível de ansiedade. Os indivíduos com níveis mais baixos de escolaridade, $9 .^{\circ}, 6 .^{\circ} \mathrm{e} 4 .^{\circ}$ anos, apresentaram maior prevalência de ansiedade leve a severa $(67,80$ e $50 \%$, respetivamente) em oposição aos dos níveis de escolaridade superiores $(0 \%$ nos doutorados, $30 \%$ nos indivíduos com mestrado, $43 \%$ nos licenciados e $47 \%$ naqueles com o $12 .^{\circ}$ ano). Não se verificou associação entre a depressão e o nível de escolaridade (Tabela 8).

Os utentes desempregados, seguidos dos trabalhadores em lay-off, foram os que apresentaram maior pre- valência de ansiedade leve a severa ( $72 \%$ e $50 \%$ respetivamente), com significância estatística (Tabela 9).

Relativamente à depressão não se verificou associação estatisticamente significativa entre esta variável e a situação profissional (Tabela 10).

Finalmente, na questão relativa ao apoio social em caso de necessidade, $89,1 \%$ consideraram ter o apoio necessário.

\section{DISCUSSÃO}

O presente estudo focou-se na prevalência das perturbações de ansiedade e depressão nos utentes da USF Santiago de Leiria durante o estado de emergência nacional por COVID-19, bem como a sua distribuição por sexo, idade, estado civil, escolaridade e situação profissional.

Os valores encontrados reforçam a dimensão do problema na população estudada. Verificou-se uma elevada prevalência quer dos sintomas de ansiedade 


\begin{tabular}{|c|c|c|c|c|c|c|c|}
\hline & & \multicolumn{5}{|c|}{ Nível de ansiedade } & \\
\hline & & Normal & Leve & Moderada & Severa & Total & \\
\hline \multirow{7}{*}{ Nível de escolaridade } & 4. ${ }^{\circ}$ Ano & 1 & 1 & 0 & 0 & 2 & \multirow{7}{*}{$p=0,015^{*}$} \\
\hline & 6. Ano & 1 & 1 & 2 & 1 & 5 & \\
\hline & 9. ${ }^{\circ}$ Ano & 14 & 6 & 16 & 6 & 42 & \\
\hline & 12. ${ }^{\circ}$ Ano & 55 & 22 & 24 & 2 & 103 & \\
\hline & Licenciatura & 62 & 24 & 21 & 2 & 109 & \\
\hline & Mestrado & 16 & 3 & 4 & 0 & 23 & \\
\hline & Doutoramento & 1 & 0 & 0 & 0 & 1 & \\
\hline \multicolumn{2}{|l|}{ Total } & 150 & 57 & 67 & 11 & 285 & \\
\hline
\end{tabular}

Legenda: ${ }^{*}=$ qui-quadrado.

\begin{tabular}{|c|c|c|c|c|c|c|c|}
\hline & & \multicolumn{5}{|c|}{ Nível de depressão } & \\
\hline & & Normal & Leve & Moderada & Severa & Total & \\
\hline \multirow{7}{*}{ Nível de escolaridade } & 4. ${ }^{\circ}$ Ano & 2 & 0 & 0 & 0 & 2 & \multirow{7}{*}{$p=0,078^{*}$} \\
\hline & 6. Ano & 3 & 0 & 2 & 0 & 5 & \\
\hline & 9. Ano & 19 & 10 & 13 & 0 & 42 & \\
\hline & 12. ${ }^{\circ}$ Ano & 73 & 16 & 12 & 2 & 103 & \\
\hline & Licenciatura & 78 & 21 & 10 & 0 & 109 & \\
\hline & Mestrado & 19 & 1 & 3 & 0 & 23 & \\
\hline & Doutoramento & 1 & 0 & 0 & 0 & 1 & \\
\hline \multicolumn{2}{|l|}{ Total } & 195 & 48 & 40 & 2 & 285 & \\
\hline
\end{tabular}

Legenda: ${ }^{*}$ = qui-quadrado.

(47\%) quer dos sintomas de depressão (32\%).

A depressão tinha, em 2017, uma prevalência de $3,44 \%$ a nível mundial, sendo mais prevalente no sexo feminino $(4,12 \%)$ do que no masculino $(2,73 \%)$. No mesmo ano, em Portugal observou-se uma prevalência de depressão na população de $4,42 \% .^{13}$

A proporção da população global com perturbações de ansiedade em 2017 foi estimada em 3,76\%. Como acontece com a depressão, estas são mais comuns entre as mulheres $(4,69 \%$ em comparação com $2,82 \%$ nos homens, a nível global). Em 2017, em Portugal obser- vou-se uma prevalência de ansiedade de 5,38\%. ${ }^{13}$

Em dezembro de 2019 estava codificada com o diagnóstico de ansiedade $7 \%$ da população adulta (idade igual ou superior a 18 anos) da USF Santiago de Leiria, maioritariamente em utentes do sexo feminino (70\%). Em relação à depressão, 13,8\% da população adulta encontrava-se codificada com este diagnóstico, sendo uma vez mais a maioria destes doentes $(78 \%)$ do sexo feminino (de acordo com os dados do MIM@UF).

Relativamente aos dados obtidos verifica-se que os indivíduos do sexo feminino apresentam níveis mais 


\begin{tabular}{|c|c|c|c|c|c|c|c|}
\hline & & \multicolumn{5}{|c|}{ Nível de ansiedade } & \\
\hline & & Normal & Leve & Moderada & Severa & Total & \\
\hline \multirow{7}{*}{ Situação profissional } & Estudante & 5 & 2 & 0 & 0 & 7 & \multirow{7}{*}{$p=0,013^{*}$} \\
\hline & Reformado & 17 & 7 & 6 & 1 & 31 & \\
\hline & Desempregado & 9 & 7 & 10 & 6 & 32 & \\
\hline & Trabalhador por conta de outrem & 109 & 38 & 44 & 4 & 195 & \\
\hline & Trabalhador-estudante & 2 & 0 & 2 & 0 & 4 & \\
\hline & Em lay-off & 0 & 1 & 1 & 0 & 2 & \\
\hline & Trabalhador por conta própria & 8 & 2 & 4 & 0 & 14 & \\
\hline \multicolumn{2}{|l|}{ Total } & 150 & 57 & 67 & 11 & 285 & \\
\hline
\end{tabular}

Legenda: ${ }^{*}=$ qui-quadrado.

\begin{tabular}{|c|c|c|c|c|c|c|c|}
\hline & & \multicolumn{5}{|c|}{ Nível de depressão } & \\
\hline & & Normal & Leve & Moderada & Severa & Total & \\
\hline \multirow{7}{*}{ Situação profissional } & Estudante & 6 & 1 & 0 & 0 & 7 & \multirow{7}{*}{$p=0,054^{*}$} \\
\hline & Reformado & 20 & 5 & 6 & 0 & 31 & \\
\hline & Desempregado & 14 & 7 & 10 & 1 & 32 & \\
\hline & Trabalhador por conta de outrem & 140 & 33 & 22 & 0 & 195 & \\
\hline & Trabalhador-estudante & 3 & 1 & 0 & 0 & 4 & \\
\hline & Em lay-off & 2 & 0 & 0 & 0 & 2 & \\
\hline & Trabalhador por conta própria & 10 & 1 & 2 & 1 & 14 & \\
\hline \multicolumn{2}{|l|}{ Total } & 195 & 48 & 40 & 11 & 285 & \\
\hline
\end{tabular}

Legenda: ${ }^{*}=$ qui-quadrado.

elevados de sintomas de ansiedade (53\%) e de depressão (36\%) comparativamente com os indivíduos do sexo masculino, mantendo a tendência pré-pandemia.

Extrapolando os dados para a população em estudo observou-se um aumento da prevalência da sintomatologia ansiosa e depressiva, que poderá dever-se à pandemia, mas também a falhas de codificação da ICPC-2, podendo os valores de 2019 estar subcodificados, sendo este um possível fator de confundimento.

Os indivíduos com níveis mais baixos de escolaridade $\left(4 .^{\circ}, 6 .^{\circ}\right.$ e $9 .^{\circ}$ anos) apresentaram maior prevalência de ansiedade. Este facto poderá relacionar-se com uma baixa literacia em saúde, o desconhecimento sobre os riscos e a gravidade da doença, assim como estratégias de coping menos eficazes.

No que diz respeito à situação profissional foram os desempregados e os trabalhadores em lay-offque apresentaram maior prevalência de ansiedade, possivelmente devido à instabilidade, à incerteza e a insegurança relacionadas com a manutenção do seu posto de trabalho ou com a necessidade de recolocação no mercado de trabalho. 
Estes dados são congruentes com outros estudos realizados durante a pandemia, em que se verificou uma maior prevalência destas psicopatologias, com maior expressão no sexo feminino. ${ }^{14-15}$

Este estudo reforça a importância da implementação de novas estratégias de prevenção em possíveis surtos subsequentes e, por outro lado, alerta os médicos para o aumento da prevalência destas patologias.

Existem algumas limitações que devem ser consideradas neste estudo. Em relação ao questionário, o facto de este ter sido de autoaplicação e realizado online poderá ter limitado o acesso a pessoas mais idosas. Por outro lado, o próprio contexto de desinformação, o desconhecimento e a pouca evidência nas informações transmitidas à população à data de aplicação poderão ter constituído um viés ao estudo.

Deste modo, os autores consideram importante a realização de estudos adicionais de seguimento, que poderão ser úteis não só na abordagem destes utentes, como no desenvolvimento de estratégias que permitam prevenir e mitigar os efeitos psicossociais da pandemia.

\section{CONCLUSÃO}

No estudo desenvolvido foram encontrados mais resultados estatisticamente significativos no estudo da ansiedade do que no estudo da depressão. Numa altura marcada pela incerteza, pela imprevisibilidade e pelo medo urge a definição de estratégias de abordagem destes utentes, de forma a criar uma maior proximidade e um ambiente que predisponha a abertura e confiança. Assim, os autores consideram fundamental que os médicos, em particular os médicos de família, estejam atentos aos seus utentes e mostrem disponibilidade para os ouvir e criem estratégias que permitam, não só o alívio dos sintomas, como a capacitação dos utentes para enfrentar esta pandemia. Aproximadamente $47 \%$ dos indivíduos apresentaram sintomas de ansiedade e $32 \%$ sintomas de depressão. O género feminino apresentou níveis de ansiedade e depressão mais elevados. Indivíduos com menor grau de escolaridade, desempregados e trabalhadores em lay-off foram os que apresentaram maior prevalência de ansiedade. Estes resultados podem ser utilizados para a definição de estratégias de melhoria da saúde mental dos utentes, nomeadamente através de um seguimento mais frequen- te dos indivíduos que referem maior sintomatologia ansiosa e depressiva, assim como dos que referem apresentar menos apoio em caso de necessidade. Como estratégia de melhoria foi reforçada a mensagem de que, apesar do confinamento, as equipas de saúde da USF Santiago de Leiria se encontravam à disposição dos seus utentes, tendo-se privilegiado o contacto através de telefone ou $e$-mail, mas sempre com a possibilidade dos doentes se dirigirem à USF quando sentiam que os seus problemas não podiam ser resolvidos através de contactos indiretos.

Numa fase em que se passou de uma situação totalmente desconhecida para uma nova normalidade, é agora necessário reavaliar a saúde mental dos utentes, nomeadamente dos mais isolados.

\section{AGRADECIMENTOS}

Os autores gostariam de agradecer à Dra. Maria de Lurdes Pereira pela leitura crítica do trabalho.

\section{REFERÊNCIAS BIBLIOGRÁFICAS}

1. Lauer SA, Grantz KH, Bi Q, Jones FK, Zheng Q, Meredith HR, et al. The incubation period of coronavirus disease 2019 (COVID-19) from publicly reported confirmed cases: estimation and application. Ann Intern Med. 2020;172(9):577-82.

2. World Health Organization. Coronavirus disease 2019 (COVID-19): situation report - 54 (14 March 2020) [homepage]. Geneva:WHO; 2020. Available from: https://apps.who.int/iris/handle/10665/331478.

3. World Health Organization. Coronavirus disease 2019 (COVID-19): situation report - 51 (11 March 2020) [homepage]. Geneva:WHO; 2020. Available from: https://apps.who.int/iris/handle/10665/331475.

4. Direção-Geral da Saúde. Casos de infeção por novo coronavírus (COVID-19): comunicado n. ${ }^{\circ}$ C160_75_v1, de 02/03/2020. Lisboa: DGS; 2020.

5. Machado RS, editor. Plano nacional de preparação e resposta à doença por novo coronavírus (COVID-19) [Internet]. Lisboa: Direção-Geral da Saúde; 2020. Available from: https://covid19.min-saude.pt/wp-content/uploads/2020/03/Plano-Nacional.pdf.

6. Decreto-Lei n. ${ }^{\circ}$ 2-A/2020, de 20 de março. Diário da República. $1^{\text {a Sé- }}$ rie(57 Suppl 1).

7. Wang C, Pan R, Wan X, Tan Y, Xu L, Ho CS, et al. Immediate psychological responses and associated factors during the initial stage of the 2019 coronavirus disease (COVID-19) epidemic among the general population in China. Int J Environ Res Public Health. 2020;17(5):1729.

8. European Center for Disease Prevention and Control. Coronavirus disease 2019 (COVID-19) pandemic: increased transmission in the EU/EEA and the UK - seventh update [homepage]. Stockholm: EDC; 2020. Available from: https://www.ecdc.europa.eu/en/publications-data/rapid-risk-assessment-coronavirus-disease-2019-covid-19-pandemic

9. Asmundson GJ, Taylor S. Coronaphobia: fear and the 2019-nCoV outbreak. J Anxiety Disord. 2020;70:102196. 
10. Brooks SK, Webster RK, Smith LE, Woodland L, Wessely S, Greenberg N, et al. The psychological impact of quarantine and how to reduce it: rapid review of the evidence. Lancet. 2020;395(10227):912-20.

11. Fernandes AC. Avaliação da eficácia de um programa de intervenção psicológica breve em pacientes pós-síndroma coronária aguda [dissertation]. Braga: Escola de Psicologia, Universidade do Minho; 2012.

12. Sousa C, Pereira MG. Morbilidade psicológica e representações da doença em pacientes com esclerose múltipla: estudo de avaliação da "Hospital Anxiety and Depression Scale" (HADS) [Psychological morbidity and illness representation in patients with multiple sclerosis: validation study of the 'Hospital Anxiety and Depression Scale' (HADS)]. Psicol Saúde Doenç. 2008;9(2):283-98. Portuguese

13. Ritchie H, Roser M. Mental health. In: Our World in Data.org [homepage]; 2018. Available from: https://ourworldindata.org/mental-health

14. Shi L, Lu ZA, Que JY, Huang XL, Liu L, Ran MS, et al. Prevalence of risk factors associated with mental health symptoms among the general population in China during the coronavirus disease 2019 pandemic. JAMA Netw Open. 2020;3(7):e2014053.
15. Solomou I, Constantinidou F. Prevalence and predictors of anxiety and depression symptoms during the covid-19 pandemic and compliance with precautionary measures: age and sex matter. Int J Environ Res Public Health. 2020;17(14):4924.

\section{CONFLITO DE INTERESSES}

Os autores declaram não possuir quaisquer conflitos de interesse.

\section{FONTES DE FINANCIAMENTO}

Os autores declaram não ter recebido quaisquer subsídios ou bolsas para a elaboração do artigo.

\section{ENDEREÇO PARA CORRESPONDÊNCIA}

Marta Marques Santana

E-mail: marquescm@hotmail.com

https://orcid.org/0000-0002-8452-0956

Recebido em 27-11-2020

Aceite para publicação em 18-07-2021

\section{ABSTRACT}

\section{ANXIETY AND DEPRESSION DURING THE NATIONAL STATE OF EMERGENCY FOR COVID-19: THE REALITY OF A FAMILY HEALTH UNIT}

Introduction: A few months have passed since the first case of COVID-19 disease in Portugal was confirmed. The rapid progression of this pandemic has led to several changes in the daily lives of the users of the Family Health Unit (FHU) Santiago de Leiria, probably with an impact on their mental health. The aim of our work is to determine the degrees of anxiety and depression of the users of the FHU Santiago de Leiria during a state of national emergency.

Study design: Cross-sectional descriptive study, with an analytical component.

Population: Users of the FHU Santiago de Leiria.

Methods: The study was carried out through an online questionnaire consisting of a sociodemographic survey, the Hospital Anxiety and Depression Scale (HADS), and a question related to social support, sent to 1,000 users of the family health unit on April 9, 2020, and valid for submission of responses until May 1, 2020.

Results: Of the 285 individuals who participated, $47 \%$ had symptoms of anxiety and $32 \%$ depressive symptoms. Individuals of the feminine gender, who had concluded high school or college, presented higher levels of both anxiety and depressive symptoms. Individuals with lower levels of education, unemployed, and lay-off workers presented with a higher prevalence of anxiety. Among respondents, $89.1 \%$ reported having the necessary social support.

Conclusion: Our work demonstrates a high prevalence of symptoms of anxiety and depression, with greater expression in females. From this premise, new strategies to improve the mental health of users should arise to prevent further damage caused by COVID-19.

Keywords: Anxiety; COVID-19; Depression; Pandemics; SARS-CoV-2. 